\title{
Spatial profiling of optical gain for optimizing lasing in plasmonic nano-lasers
}

\author{
Z. A. Sattar \\ z.a.sattar@bangor.ac.uk \\ K. A. Shore
}

School of Electronic Engineering, Bangor University Bangor, Wales LL57-1UT, UK

School of Electronic Engineering, Bangor University Bangor, Wales LL57-1UT, UK

Design of cylindrical metal-clad semiconductor nano-lasers is undertaken. Specific attention is given to determining the modal gain in structures supporting $\mathrm{TM}_{01}$ Surface Plasmon Polarition (SPP) modes. For representative structures it is indicated that cavity lengths of order $100 \mu \mathrm{m}$ enable lasing action. In comparison structures supporting $\mathrm{TE}_{01}$ core-confined modes having cavity lengths of order $10 \mu \mathrm{m}$ may sustain lasing. The analysis methodology adopted offers means to affect the design of candidate semiconductor lasers for bespoke applications.

[DOI: http://dx.doi.org/10.2971/jeos.2013.13045]

Keywords: Inner core, lasing, material gain, metal-clad, modal gain, nano-laser, outer core

\section{INTRODUCTION}

Driven by potential applications in photonic integrated circuits, optical information processing and system-on-a-chip technologies considerable effort has been directed at developing sub-wavelength nano-scale semiconductor lasers. This work led to the development of novel geometries [1]-[5]. Metal-clad semiconductor lasers offer significant for realizing the potential of nano-scale lasers. A variety of such structures have been explored in recent years [6]-[13], including FabryPerot lasers [14], nanowire lasers [15], nanopatch lasers [16], and spaser-based nano- lasers [17].

A specific class of such devices which has attracted interest is cylindrical metal-clad nano-lasers for which attention has been given to their operation based on the excitation and support of Surface Plasmon Polariton (SPP) waveguide modes [18]-[21].

Vivek et al. investigated the effect of metal cladding on the coupling of SPP modes emphasizing their mode confinement and loss characteristics [20]. A recent study by Ikeda et al. gives insight to the gain properties of the structure [21], where it is assumed that the active core region could be modelled as a single layer of prescribed optical properties and specifically of defined optical gain.

Such nano-lasers are anticipated to exhibit enhanced dynamical performance which may arise from a combination of physical factors including enhanced modal refractive indices and Purcell cavity-enhanced spontaneous emission. However in complementary work [22] on the dynamical performance of metal-clad nano-lasers it was shown by means of a simple analysis that the direct-current modulation bandwidth of such lasers may suffer deleterious effects due to those factors.
In that context, the aim of the present work is to explore the opportunities and challenges which arise in the design of metal-clad semiconductor lasers giving specific attention to the role of SPP modes. The approach adopted is to take into account spatial profiling of the optical gain as a means both for optimizing lasing operation and as a step towards a fully self-consistent theoretical model of such structures.

The paper is organized as follows. Firstly, a description of the laser structure is given and then the method used to effect optical mode analysis for the structure is outlined. Results using the methodology are presented. Finally, conclusions are drawn based on the results obtained.

\section{LASER STRUCTURE AND MODEL}

The structure under consideration is a cylindrical metal-clad nano-laser. In previous work such a structure has been studied assuming a uniform core [20, 21]. In order to take into account spatial variations in gain in the active region of such a structure a two-layer active core model is utilized. The crosssection of such a structure is illustrated in Figure 1. The key elements of the structure are the core semiconductor region and the metal cladding. The analysis methodology adopted here is of general application but for the work presented here it is assumed that the metal-clad structure is surrounded by air. In addition, for all results discussed in this paper the operating wavelength of the laser is taken to be $1 \mu \mathrm{m}$. It is straightforward to examine alternative configurations and notably the use of alternative surrounding media. Following earlier work the core semiconductor material is assumed to be $\operatorname{In}_{0.2} \mathrm{Ga}_{0.8}$ As $[23,24]$ with a refractive index of 3.6 at the chosen operating wavelength. The metal cladding is taken to be gold with a 


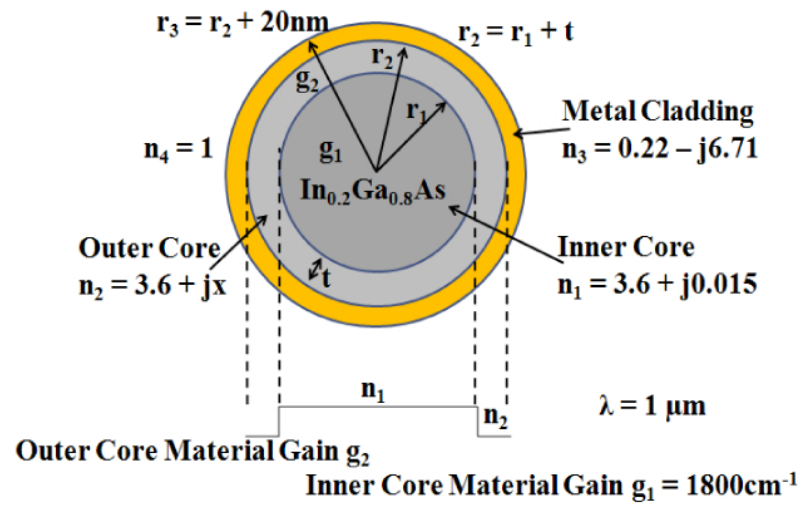

FIG. 1 Cross-section of cylindrical semiconductor metal-clad nano-laser.

complex refractive index of $0.22-j 6.71$ [25] and the thickness of metal clad is assumed to be $20 \mathrm{~nm}$ [26].

The model which has been developed for this work allows for the definition of an arbitrary number of layers in the active layer core semiconductor region as well as in the cladding region. This utility has been developed in order to explore combinations of gain profiles and/or surrounding media.

However, for the specific calculations performed here, the core semiconductor is considered to be comprised of just two regions with differing material gains. In this case, the overall core radius $r_{2}$ is defined as the sum of the radius of the 'inner core', $r_{1}$, and the thickness of the 'outer core', $t$.

For the cases explored here the gain in the inner core, $g_{1}$ is taken to be greater than that of the outer core $g_{2}$. It is underlined that the relative values of such gains are dependent upon the precise electrical excitation scheme utilised in such lasers. The methodology allows exploration of such alternatives. For the sake of definiteness, the results obtained here have sought to make a comparison of the lasing conditions when allowance is made for a variation in the outer core material gain $g_{2}$ whilst the inner core material gain $g_{1}$ is held at a fixed value of $1800 \mathrm{~cm}^{-1}$.

The material gain, $g_{1}$ in the inner core is introduced through the imaginary part of the refractive index of the active region, which is related to the gain coefficient by $\frac{4 \pi}{\lambda} \operatorname{Im}\left(n_{\text {core }}+j k_{\text {core }}\right)$. Where $n_{\text {core }}$ is real part of the refractive index and $k_{\text {core }}$ is the imaginary part of the refractive index. The real part of the inner core region is kept constant to 3.6 whereas the gain is introduced by varying the imaginary part from 0 to $j 0.015$ [21]. This in turns provide a maximum material gain in the inner core which approximates to $1800 \mathrm{~cm}^{-1}$ at the wavelength of $1 \mu \mathrm{m}$. In effect, when the outer core layer was introduced in the core region, the material gain $g_{1}$ was kept constant to $1800 \mathrm{~cm}^{-1}$ and $g_{2}$ was varied from 0 to $1760 \mathrm{~cm}^{-1}$ which is equivalent to a refractive index of the outer core $3.6+j 0.014$. The model developed here provides the basis for more detailed nano-laser design and specifically is capable of extension to provide a self-consistent analysis of the wave-guiding and lasing properties of such metal-clad cylindrical nano-lasers.

Lasers constructed with such geometries may support several kinds of waveguide modes and notably bound modes which are largely confined to the active core and surface Plasmon polariton (SPP) modes which derive from the dielectric-metal interface. In this work attention has been given to both kinds of modes and notably confined TE modes and low order TM SPP modes.

\section{ANALYSIS TECHNIQUE}

Analysis of the structure has been performed using the wellknown transfer matrix method [4], [27] which, in principle, enables direct evaluation of structures where consideration is given to an arbitrary number of multilayers. The outline of the analysis technique presented here is specifically for the case under consideration i.e. with a two-layer active core; a metal cladding and the surrounding medium being air. It is noted that some algebraic manipulation needs to be undertaken when the number of layers utilised is changed.

In order to evaluate the gain properties for the two-layer active core metal-clad cylindrical structure of Figure 1, the relevant optical modes need to be obtained. We consider $\mathrm{z}$ axis as the direction of propagation and therefore, every field component within the cylindrical structure can be represented in the form,

$$
\Phi(r, \psi, z, t)=\Phi(r, \psi) e^{j(\beta z-\omega t)}
$$

Where $\Phi$ represents the field components in the structure, i.e. $E_{z}, E_{r}, E_{\theta}, H_{z}, H_{r}, H_{\theta} . \omega$ being the angular frequency and $\beta$ is the propagation constant. For the analysis of the structure, the transverse field component for the TM and TE mode is expressed in terms of $E_{z}$ and $H_{z}$ respectively,

$$
\begin{gathered}
E_{z i}=\left[P_{i} J_{o}\left(\gamma_{i} r_{i}\right)+Q_{i} Y_{o}\left(\gamma_{i} r_{i}\right)\right] \\
H_{z i}=\left[M_{i} J_{o}\left(\gamma_{i} r_{i}\right)+N_{i} Y_{o}\left(\gamma_{i} r_{i}\right)\right]
\end{gathered}
$$

Where $P_{i}, Q_{i}, M_{i}, N_{i}$, are constants; $i$ represents the appropriate region of the structure. For the case under consideration $i=1,2,3,4$ representing the inner core, outer core, metal cladding and outermost dielectric, $J_{0}$ and $Y_{0}$ are the Bessel functions of the first and the second kind respectively and $\gamma_{i}$ is the propagation constant in each layer which is defined by;

$$
\gamma_{i}=\left[(\omega / c)^{2} n_{i}^{2}-\beta^{2}\right]^{1 / 2}
$$

Following the standard matrix approach the boundary conditions are satisfied by using matrix, $X$ and $Y$, which links the field parameters of adjacent layers i.e. $P_{i}, Q_{i}, M_{i}, N_{i}$, to $P_{i+1}, Q_{i+1}, M_{i+1}, N_{i+1}$, for the TM and TE modes respectively. Final Matrix for the TM \& TE modes that links the field parameters from the first layer i.e. inner core to the outer most cladding i.e. air, can be represented as,

For TM mode,

$$
\left(\begin{array}{l}
\mathbf{P}_{4} \\
\mathbf{Q}_{4}
\end{array}\right)=\mathbf{X}\left(\begin{array}{l}
\mathbf{P}_{1} \\
\mathbf{Q}_{1}
\end{array}\right)
$$

For TE mode,

$$
\left(\begin{array}{l}
\mathbf{M}_{4} \\
\mathbf{N}_{4}
\end{array}\right)=\mathbf{Y}\left(\begin{array}{l}
\mathbf{M}_{1} \\
\mathbf{N}_{1}
\end{array}\right)
$$


In order to obtain a finite field at the centre of the core and a decaying field in the outermost dielectric we require,

$$
Q_{1}=0, N_{1}=0, Q_{4}=j P_{4} \quad \text { and } \quad N_{4}=j M_{4}
$$

The elements of $X$ and $Y$ are relatively involved expressions and are not re-produced here. Using Eq. (7) in Eq. (5) and Eq. (6), leads to an eigenvalue matrix in the form:

For TM mode,

$$
\left(\begin{array}{ll}
X_{11} & -1 \\
X_{21} & -j
\end{array}\right)\left(\begin{array}{l}
P_{1} \\
P_{4}
\end{array}\right)=0
$$

For TE mode,

$$
\left(\begin{array}{ll}
Y_{11} & -1 \\
Y_{21} & -j
\end{array}\right)\left(\begin{array}{l}
M_{1} \\
M_{4}
\end{array}\right)=0
$$

Setting the determinant of the matrix in Eq. (8) and (9) to zero, we have:

For TM;

$$
X_{21}-j X_{11}=0
$$

For TE;

$$
Y_{21}-j Y_{11}=0
$$

The eigenvalue equations for the TM and TE modes were then used to evaluate the effective refractive index of the relevant modes. In this way the optical gain of the TM and TE modes is found and hence the lasing condition for the structure is determined. Simulations were performed using MATLAB.

\section{RESULTS}

The principal aim of this paper is to evaluate the optical gain and lasing condition for the TM SPP mode which can be supported by the structure of interest. However, in order to bench mark the laser performance in that mode we have also examined the operating conditions for the low order $\mathrm{TE}_{01}$ mode which also has the potential to lase in the chosen structure. The lasing condition depends on the modal gain and the reflectivity of the laser. In general the lasing condition is defined by the length of the device $L$ as shown in Figure 2.

$$
L=\frac{1}{G} \ln \left(\frac{1}{R}\right)
$$

Where, $G$ is the modal gain and $R$ is the reflectivity of the laser.

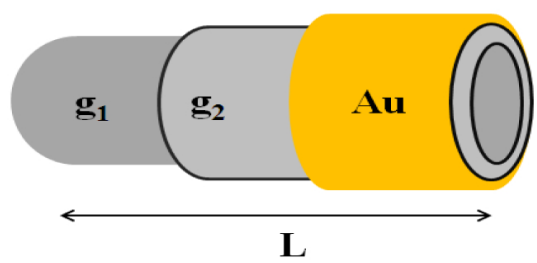

FIG. 2 Representation for the Length ' $L$ ' of the device.

\subsection{TE 01 mode analysis}

In the analysis the dimensions of the device were fixed in the following way. The overall core radius $\left(r_{2}\right)$ was held constant at $200 \mathrm{~nm}$ whilst the inner core radius $\left(r_{1}\right)$ and outer core thickness $(t)$ were varied. The analysis is performed for different inner core radius and outer core thickness and a comparison is drawn with respect to the modal gain.

Calculations are therefore done for the structure without outer core, i.e. $r_{1}=r_{2}=200 \mathrm{~nm}$, and with outer core, as summarized in Table 1. Outcomes of representative calculations where the inner core and outer core material gain is varied are shown in Figure 3. It is observed that the modal gain linearly increases as the material gain is increased. For the dual core structure with the inner core material gain of $1800 \mathrm{~cm}^{-1}$ and variation in the outer core material gain we observe a shift in the modal gain towards the positive at zero material gain for the dual core structure. Furthermore, an increase in the inner core radius shifts the modal gain towards the more positive end. The shift in modal gain also depends on the thickness of the outer core.

Having obtained the appropriate modal gain it is possible to

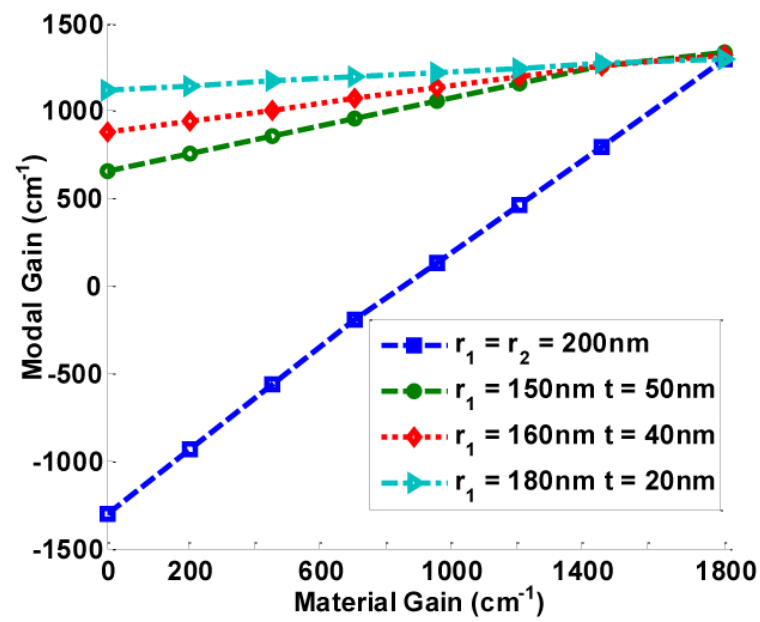

FIG. $3 \mathrm{TE}_{01}$ Modal Gain vs Material Gain for different inner core radii for fixed overall core radius.

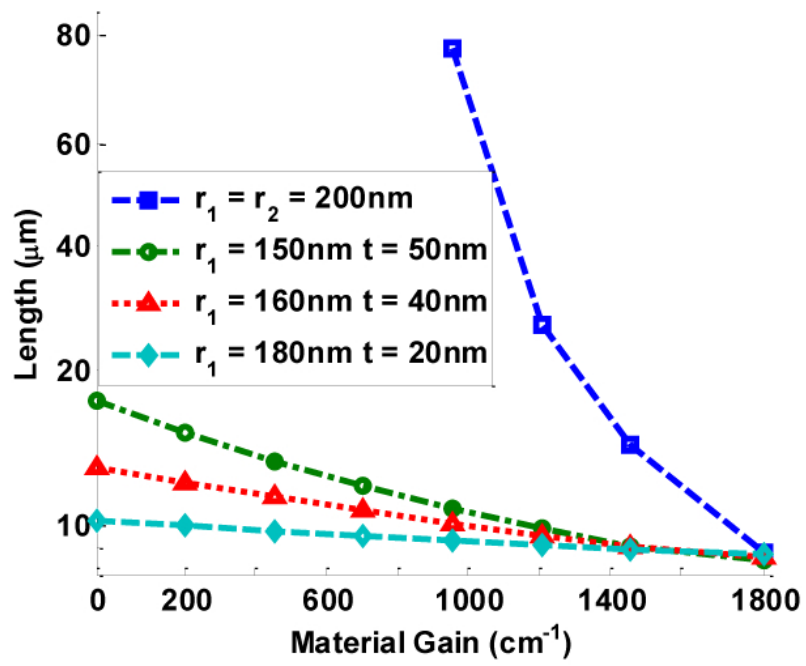

FIG. 4 Device Length versus Material Gain for $\mathrm{TE}_{01}$ mode. 


\begin{tabular}{|c|c|c|c|c|}
\hline $\begin{array}{c}\text { Inner Core } \\
\text { radius } r_{1}(\mathrm{~nm})\end{array}$ & $\begin{array}{c}\text { Outer Core } \\
\text { thickness } \mathrm{t}(\mathrm{nm})\end{array}$ & $\begin{array}{c}g_{1} \\
\left(\mathrm{~cm}^{-1}\right)\end{array}$ & $\begin{array}{c}g_{2} \\
\left(\mathrm{~cm}^{-1}\right)\end{array}$ & $\begin{array}{c}\text { Length } \\
(\mu \mathrm{m})\end{array}$ \\
\hline 200 & - & $0-1800$ & - & $9-80$ \\
\hline 150 & 50 & 1800 & $0-1760$ & $9-18$ \\
\hline 160 & 40 & 1800 & $0-1760$ & $9-15$ \\
\hline 180 & 20 & 1800 & $0-1760$ & $9-10$ \\
\hline
\end{tabular}

TABLE 1 Specifications for the $T E_{01}$ mode analysis

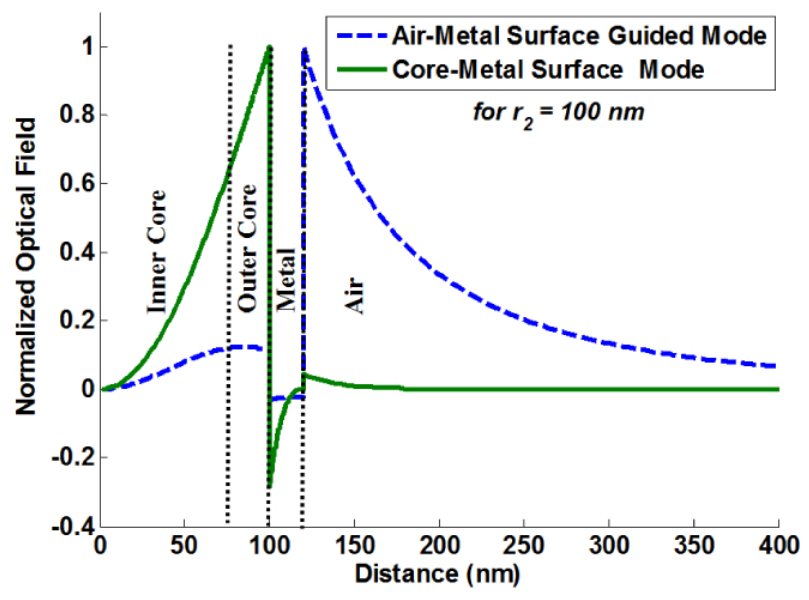

FIG. 5 Optical Field of the $\mathrm{TM}_{01}$ Air-Metal and Core-Metal surface guided modes.

utilise the standard lasing condition to determine the length of device required to achieve lasing. Outcomes of such calculations are given in Figure 4. It is noted that devices supporting $\mathrm{TE}_{01}$ modes having cavity lengths of order $10 \mu \mathrm{m}$ appear to have the potential to support lasing action.

\subsection{TM01 SPP mode analysis}

Attention is now turned to the modal gain and lasing conditions for the SPP modes of the defined structure. In this respect the capability of the present model to examine profiled optical gain will be exploited to identify conditions under which lasing action may be achieved. We may identify two kinds of SPP modes: asymmetric SPP modes (also known as core-metal surface guided modes) and symmetric SPP modes (also known as air-metal surface guided modes) [18], [28]. The modes guided at the air-metal interface are known as long range surface plasmon modes which are of much practical interest in cylindrical devices that incorporate small core radius. On the other hand, core-metal surface guided modes experience high losses [20] and may have reduced applications potential.

Figure 5 shows the optical field of these two types of SPP modes in the device having an overall core radius of 100 $\mathrm{nm}$ and a metal cladding thickness of $20 \mathrm{~nm}$. The $\mathrm{TM}_{01}$ air metal surface guided mode is transformed to core guided mode when the overall core radius increases from $100 \mathrm{~nm}$ to $r_{2} \geq 220 \mathrm{~nm}$. Figure 6 shows the optical field of the TE and TM (core guided) modes formed in metal-clad cylindrical structures of radii $220 \mathrm{~nm}$. Representations of the intensity profiles of the optical modes are offered in Figure 7(a)-(c) for the $\mathrm{TM}_{01}$ air-metal, core-metal surface guided and core guided modes and $7(\mathrm{~d}) \mathrm{TE}_{01}$ mode, which corresponds to Figure 5 and Fig-

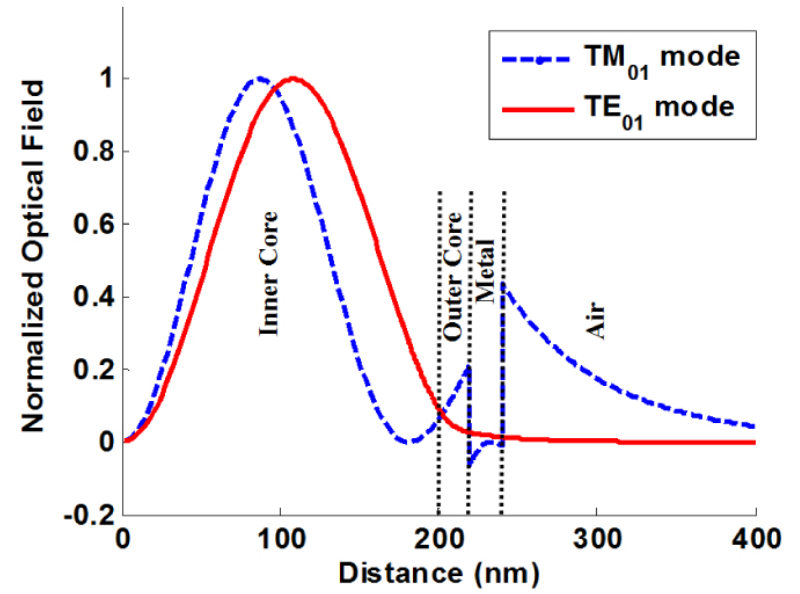

FIG. 6 Optical Field of the $\mathrm{TM}_{01}$ Core-Guided mode and $\mathrm{TE}_{01}$ mode at Core radius of 220 $\mathrm{nm}$. (a)

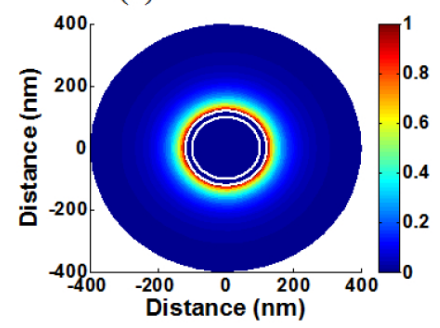

(c)

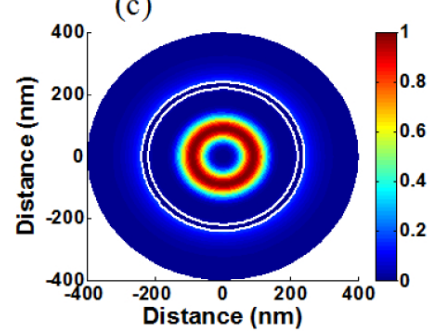

(b)

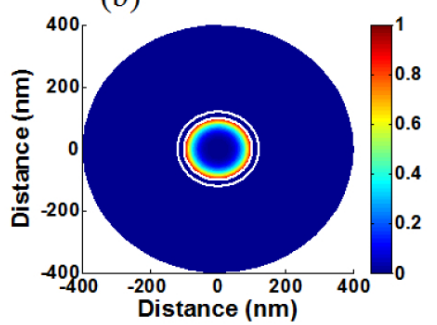

(d)

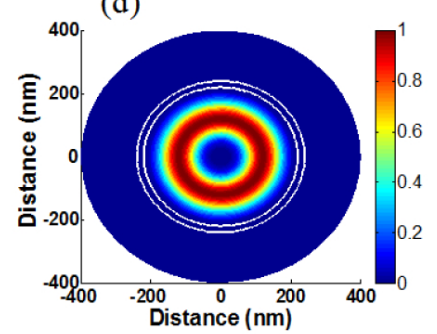

FIG. 7 Intensity profile of the (a) $\mathrm{TM}_{01}$ Air-Metal surface guided mode (b) $\mathrm{TM}_{01}$ CoreMetal surface guided mode $(c) \mathrm{TM}_{01}$ Core guided mode (d) $\mathrm{TE}_{01}$ mode.

ure 6 . In the figures the locations of the metal cladding is denoted by a double line.

As already indicated the focus of these investigations is on the opportunities offered by local gain profiling to obtain lasing action and thence to make a comparison with that obtained in the case of the TE mode analysed in the previous sub-section. As exemplars of this approach the inner core gain is held constant at $1800 \mathrm{~cm}^{-1}$ and variations allowed in the outer core material gain. Attention is also given to variations in the ra- 


\begin{tabular}{|c|c|c|c|c|}
\hline $\begin{array}{c}\text { Inner Core } \\
\text { radius } r_{1}(\mathrm{~nm})\end{array}$ & $\begin{array}{c}\text { Outer Core } \\
\text { thickness } \mathrm{t}(\mathrm{nm})\end{array}$ & $\begin{array}{c}g_{1} \\
\left(\mathrm{~cm}^{-1}\right)\end{array}$ & $\begin{array}{c}g_{2} \\
\left(\mathrm{~cm}^{-1}\right)\end{array}$ & $\begin{array}{c}\text { Length } \\
(\mu \mathrm{m})\end{array}$ \\
\hline 100 & - & $0-1800$ & - & $100-1100$ \\
\hline 50 & 50 & 1800 & $0-1760$ & $100-250$ \\
\hline 60 & 40 & 1800 & $0-1760$ & $90-150$ \\
\hline 80 & 20 & 1800 & $0-1760$ & $90-100$ \\
\hline
\end{tabular}

TABLE 2 Specifications for the $T M_{01}$ mode analysis
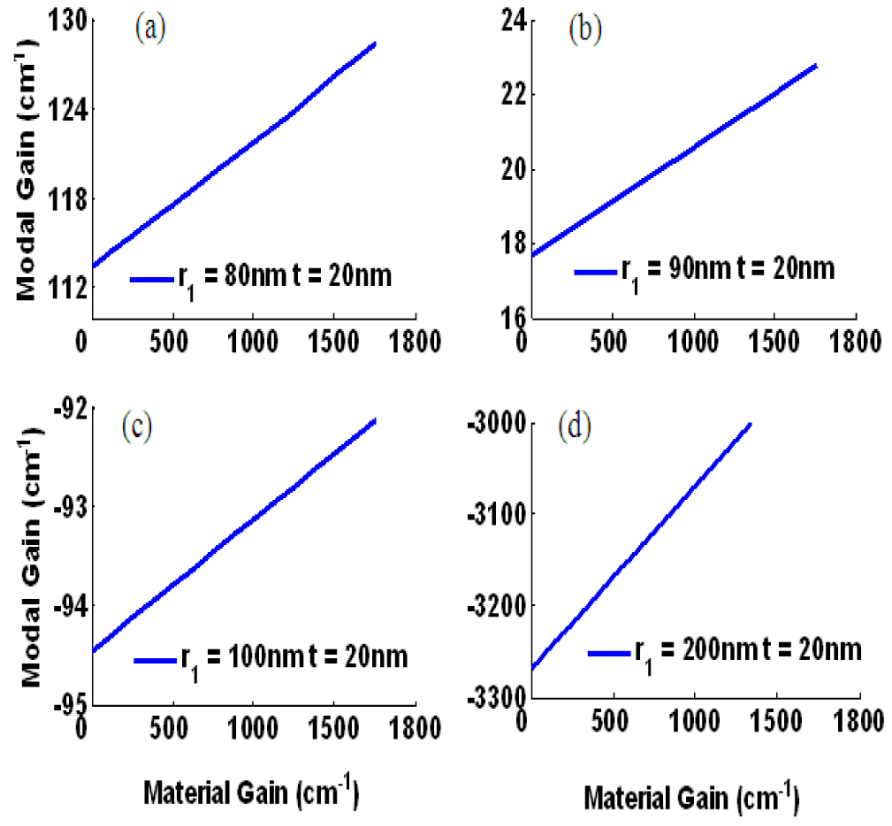

FIG. 8 (a-d) TM $_{01}$ Modal Gain versus Material Gain for different active region radii

dius of the inner core and thickness of outer core as summarized in Table 2.

Figure 8(a) shows that for a device with an inner core radius of $80 \mathrm{~nm}$ the opportunity exists to achieve a positive modal gain over a quite wide range of outer-core material gain. Figure $8(\mathrm{~b})$ through to $8(\mathrm{~d})$ show that when the inner-core radius is increased the accessible modal gain drops quite significantly such that when the inner core radius is $100 \mathrm{~nm}$ the metal losses are such that positive modal gain cannot be obtained even for large values of outer-core material gain.

It is pointed out that for the case of an inner core radius of 220nm shown in Figure 8(d) the mode is transformed into a core guided mode. Nevertheless due to the dominance of the metal losses the modal loss becomes exceedingly large.

Having established the propensity of small radius structures to deliver positive modal gain, attention is given to the influence of varying the gain profile via changes in the inner and outer core radii. In the calculations illustrated in Figure 9, the overall active core radius is set at $100 \mathrm{~nm}$ whilst the values of the inner and outer core dimensions are changed.

Determination of the modal gain is again effected for a fixed inner core gain of $1800 \mathrm{~cm}^{-1}$ and variations in the outer core material gain allowed in comparison to a structure without outer core. As may be anticipated, when the mode is enabled

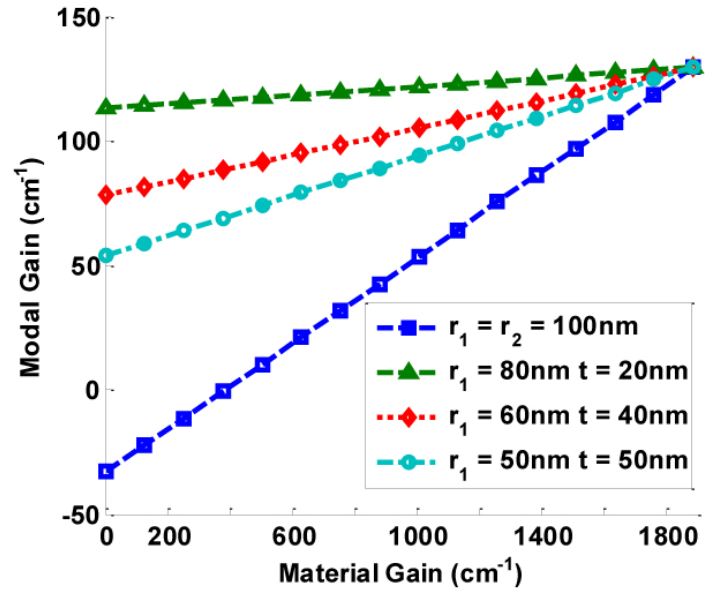

FIG. $9 \mathrm{TM}_{01}$ Modal Gain vs Material Gain for different inner core radius where the overall core radius is kept constant.

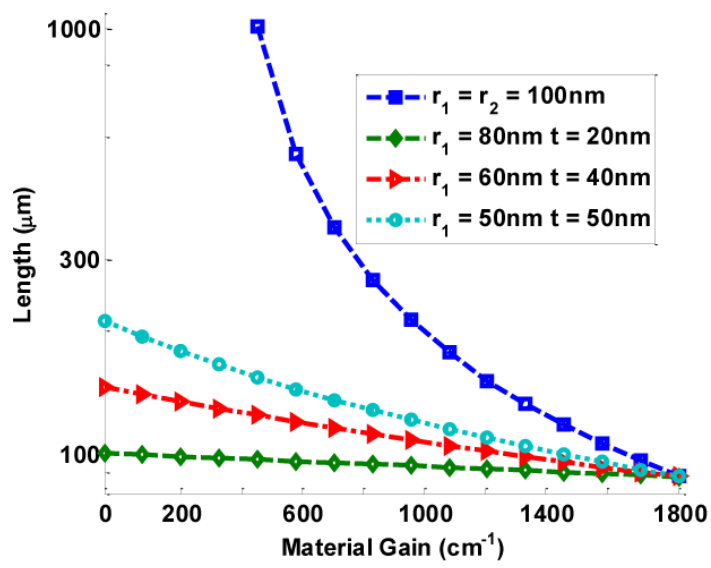

FIC. 10 Device Length vs Material Gain for $\mathrm{TM}_{01}$ mode.

to access an increased active volume of higher gain the modal gain is increased. It is noted that in all cases it is possible to achieve positive modal gain.

Guided by the results displayed in Figure 9, attention is now given to the determination of the cavity lengths required to sustain lasing. Outcomes of such calculations are shown in Figure 10. It is seen that for the TM mode significantly greater cavity lengths - here of order $100 \mu \mathrm{m}$ - are required relative to those obtained in the TE case as shown in Figure 4.

\section{CONCLUSION}

An analysis has been performed of the wave-guiding at lasing conditions in a cylindrical nano-laser structure. The model which has been utilized for this purpose allows for variations 
in the material gain of the active core to be taken into account. The model has been applied to the specific case of a two-layer active core and a comparison has been made of the requirements for achieving lasing via the excitation of either $\mathrm{TE}_{01}$ or $\mathrm{TM}_{01}$ modes. In the former case it is found that structures of length of order $10 \mu \mathrm{m}$ have the potential to lase whereas in the latter case device lengths of order $100 \mu \mathrm{m}$ are needed to achieve lasing. The model developed here provides the basis for more detailed nano-laser design and specifically is capable of extension to provide a self-consistent analysis of the waveguiding and lasing properties of such metal-clad cylindrical nano-lasers.

\section{ACKNOWLEDGEMENT}

This research work was supported from Bangor University, School of Electronic Engineering, Wales, UK and the Memon Communities in Pakistan.

\section{References}

[1] T. Baba, "Photonic crystals and microdisk cavities based on GalnAsP-InP system," IEEE J. Quantum Elect. 3, 808-830 (1997).

[2] J. Topolancik, S. Chakravarty, P. Bhattacharya, and S. Chakrabarti, "Electrically injected quantum-dot photonic crystal microcavity light sources," Opt. Lett. 31, 232-234 (2006).

[3] J. Pauzauskie, D. J. Sirbuly, and P. Yang, "Semiconductor nanowire ring resonator laser," Phys. Rev. Lett. 96, 1439031-1439034 (2006).

[4] P. Yeh, Optical waves in layered media (John Wiley a Sons, New York, 1988).

[5] D. K. Armani, T. J. Kippenberg, S. M. Spillane, and K. J. Vahala, "Ultra-high- $Q$ toroid microcavity on a chip," Nature 421, 925-928 (2003).

[6] 0. Hess, J. B. Pendry, S. A. Maier, R. F. Oulton, J. M. Hamm, and K. L. Tsakmakidis, "Active nanoplasmonic metamaterials," Nat. Mater. 11, 573-584 (2012).

[7] 0. Hess, and K. L. Tsakmakidis, "Metamaterials with quantum gain," Science 339, 654-655 (2013).

[8] A. D. Boardman, V. V. Grimalsky, Y. S. Kivshar, S. V. Koshevaya, M. Lapine, N. M. Litchinitser, V. N. Malnev, et al., "Active and tunable metamaterials," Laser Photonics Rev. 5, 287-307 (2011).

[9] C. M. Soukoulis, and M. Wegener, "Past achievements and future challenges in the development of three-dimensional photonic metamaterials," Nat. Photonics 5, 523-530 (2011).

[10] S. Wuestner, A. Pusch, K. L. Tsakmakidis, J. M. Hamm, and 0. Hess, "Overcoming losses with gain in a negative refractive index metamaterial," Phys. Rev. Lett. 105, 127401-127404 (2010).

[11] 0. Hess, S. Wuestner, A. Pusch, K. L. Tsakmakidis, and J. M. Hamm, "Gain and plasmon dynamics in active negative-index metamaterials," Philos. T. R. Soc. A 369, 3525-3550 (2011).

[12] J. M. Hamm, S. Wuestner, K. L. Tsakmakidis, and 0. Hess, "Theory of light amplification in active fishnet metamaterials," Phys. Rev. Lett. 107, 167405-167409 (2011).
[13] A. Pusch, S. Wuestner, J. M. Hamm, K. L. Tsakmakidis, and 0. Hess, "Coherent amplification and noise in gain-enhanced nanoplasmonic metamaterials: A Maxwell-Bloch Langevin approach," ACS Nano 6, 2420-2431 (2012).

[14] M. T. Hill, M. Marell, E. S. P. Leong, B. Smalbrugge, Y. Zhu, M. Sun, P. J. an Veldhoven, et al., "Lasing in metal-insulatormetal sub-wavelength plasmonics waveguides," Opt. Express 17, 11107-11112 (2009).

[15] R. F. Oulton, V. J. Sorger, T. Zentgraf, R. M. Ma, C. Gladden, L. Dai, G. Bartal, and X. Zhang, "Plasmon lasers at deep subwavelength scale," Nature 461, 629-632 (2009).

[16] K. Yu, A. Lakhani, and M. C. Wu, "Subwavelength metal-optic semiconductor nanopatch lasers," Opt. Express 18, 8790-8799 (2010).

[17] M. A. Noginov, G. Zhu, A. M. Belgrave, R. Bakker, V. M. Shalaev, E. E. Narimanov, S. Stout, et al., "Demonstration of a spaser-based nanolaser," Nature 460, 1110-1113 (2009).

[18] S. J. Al-Bader, and M. Imtaar, "Azimuthally Uniform Surface-Plasma Modes in Thin Metallic Cylindrical Shells," IEEE J. Quantum Elect. 28, 525-533 (1992).

[19] A. V. Maslov, and C. Z. Ning, "Size reduction of a semiconductor nanowire laser by using metal coating," Proc. SPIE. 6468, 646801646807 (2007).

[20] V. Krishnamurthy, and B. Klein, "Theoretical investigation of metal cladding for nanowire and cylindrical micropost lasers," IEEE J. Quantum Elect. 44, 67-74 (2008).

[21] K. Ikeda, Y. Fainman, K. A.Shore, and H. Kawaguchi, "Modified long-range surface plasmon polariton modes for laser nanoresonators." J. Appl. Phys. 110, 0631061-0631066 (2011).

[22] K. A. Shore, "Modulation bandwidth of metal-clad semiconductor nano-lasers with cavity-enhanced spontaneous emission," Electron. Lett. 46, 1688-1689 (2010).

[23] J. Noborosika, J. Motohisa, J. Takeda, M. Inari, Y. Miyoshi, N. Ooike, and T. Fukui, "Growth of GaAs and InGaAs nanowires by utilizing selective area MOVPE," in Proceedings to $16^{\text {th }}$ Int. Conf. Indium Phospide and Related Materials, (IEEE, Japan, 2004).

[24] Y. Kim, H. J. Joyce, Q. Gao, H. H. Tan, C. Jagadish, M. Paladugu, J.Zou, and A. A. Suvorova, "Influence of nanowire density on the shape and optical properties of ternary InGaAs nanowires," Nano Lett: 6, 599-604 (2006).

[25] M. A. Ordal, L. L. Long, R. J. Bell, S. E. Bell, R. R. Bell, R. W. Alexander, Jr., and C. A. Ward, "Optical properties of metals Al, Co, Cu, $\mathrm{Au}, \mathrm{Fe}, \mathrm{Pb}, \mathrm{Ni}, \mathrm{Pd}, \mathrm{Pt}, \mathrm{Ag}, \mathrm{Ti}$ and $\mathrm{W}$ in infrared and far infrared," Appl. Opt. 22, 1099-1119 (1983).

[26] G. Winter, S. Wedge, and W. L. Barnes, “Can lasing at visible wavelengths be achieved using low-loss long-range surface plasmonpolariton mode?" New J. Phys. 8, 1-14 (2006).

[27] P. Yeh, A. Yariv, and E. Marom, "Theory of Bragg fiber," J. Opt. Soc. Am. 68, 1196-1201 (1978).

[28] J. J. Burke, G. I. Stegeman, and T. Tamir, "Surface-polariton-like waves guided by thin, lossy metal films," Phys. Rev. B. 33, 5186-5201 (1986). 